\title{
Accuracy of Screening Methods for the Diagnosis of Breast Disease
}

\author{
ISOBEL G. FURNIVAL, ${ }^{*}$ M.B., CH.B. ; HELEN J. STEWART,† M.B., F.R.C.S.ED. ; J. M. WEDDELL, $\ddagger$ M.B., B.S. \\ P. DOVEY, $\$ M.B., F.F.R.; I. H. GRAVELLE, $\|$ M.R.C.P.ED., F.F.R. ; K. T. EVANS, $\$ M.R.C.P., F.F.R. \\ A. P. M. FORREST, ${ }^{* *}$ M.D., CH.M., F.R.C.S.(ENG.,ED.,GLASG.)
}

\begin{abstract}
Cummary: Clinical examination, thermography, and 70-mm. mammography were performed in 891 patients -414 presented to hospital with symptoms of breast disease and 477 were asymptomatic. Comparison of the diagnostic accuracy of these methods showed that neither thermography nor $70-\mathrm{mm}$. mammography has a useful place as an isolated screening procedure for breast cancer. In fact, we consider such a policy dangerous.
\end{abstract}

\section{Introduction}

Recently there has been increasing interest in the development of mass screening techniques for the early diagnosis of carcinoma of the breast. The most significant study from New York has been designed to compare the mortality from breast cancer in women in whom regular annual screening has been carried out by clinical examination and mammography with the mortality of those who are receiving normal medical care (Strax et al., 1967, 1968, 1969; Venet et al., 1969). Though the final evaluation of this study must await assessment of mortality, it is already apparent that those cases of breast cancer which have been discovered in the screened group have a lower incidence of lymphatic involvement than those arising in the control population. It is also clear that both methods of examination are required for satisfactory screening and that clinical examination or mammography alone detected only about half of the total number of cancers discovered.

Mammography, as ordinarily performed, uses large film and the radiographic image is the same size as the patient's breast. It is time-consuming and expensive. Alternative simpler diagnostic methods which might be suitable for screening are therefore being studied. Two such methods, thermography and 70-mm. mammography (Strax and Oppenheim, 1968; Gravelle, 1969), are the subject of this report, in which their diagnostic value has been compared with that of routine clinical examination in 891 patients with or without symptomatic breast disease.

\section{Methods}

Of the 891 patients included in the diagnostic survey, 414 (aged 16-78 years) had come to hospital with symptoms of breast disease and 477 (aged 18-78 years) had no such symptoms and were attending hospital for some unrelated reason, usually gynaecological. The survey included clinical examination, thermography, and $70-\mathrm{mm}$. mammography.

Each patient was briefly interviewed for relevant history. The breasts were then clinically examined by one observer (either I.G.F. or H.J.S.). Thermography was then performed by technicians using an AGA Thermovision apparatus. After the removal of clothes above the waist, the patient sat with hands

\footnotetext{
Clinical Research Assistant, Welsh National School of Medicine, Cardiff CF2 1 SZ. t Honorary Assistant Lecturer in Surgery, Welsh National School of
Medicine, Cardiff CF2 1SZ.

\$ Senior Research Fellow, Epidemiological Research Unit, Cardiff.

Consultant Radiologist, Royal Infirmary, Cardiff.

Professor of Radiology, Welsh National School of Medicine, Cardiff CF2 isZ.

** Professor of Surgery, Welsh National School of Medicine, Cardiff
}

on hips in the thermography room for 15 minutes. This was to allow cooling of the breasts and axillae to the temperature of the room, which was maintained between 18 and $21^{\circ} \mathrm{C}$. The hands were raised above the head and the breasts were scanned individually with the infrared camera. Frontal and oblique views of both breasts were taken and the thermographic image obtained on the oscilloscope screen was recorded on $35-\mathrm{mm}$. panchromatic film. Then $70-\mathrm{mm}$. mammography was carried out on an Odelca photofluorographic unit. This unit produces a $70-\mathrm{mm}$. photograph of the fluorographic image of the breast. Two standard views (craniocaudal and mediolateral) were taken of both breasts. As the fine-focus $x$-ray tube and an Odelca camera unit were mounted on a motorized stand, the position of the tube could be rapidly changed while the patient remained stationary in the erect position. In this way complete radiographic examination of both breasts could be carried out in five minutes. It took about 40 minutes to survey each patient by all three methods.

The results of each form of investigation were separately and independently reported. For thermography and 70-mm. mammography independent reports were received from three radiologists, each of whom examined the thermography and mammography films separately without knowledge of the patient or her complaints. As all films were processed in batches there was no opportunity for repeat examination should the films be unsatisfactory.

The patients with symptomatic disease had also been examined by a consultant surgeon. Further, all patients with symptomatic breast disease and most of those in the control series had full-size mammography, an examination which forms part of our routine assessment of patients with breast complaints.

The value of clinical examination, thermography, and 70$\mathrm{mm}$. mammography was compared by the percentage of correct diagnosis in each group of subjects.

\section{Results}

\section{Symptomatic patients}

Of the 414 women in this group 214 had histologically proved benign or malignant breast disease. A further eight women with advanced cancer did not have histological confirmation of their disease, but as the diagnosis was not in doubt they have been included with the malignant group. Seventyseven patients had cancer and 145 had proved benign disease. The remaining 192 patients in the symptomatic group had not had a biopsy performed and were considered by the clinician responsible for their care to have either benign diffuse disease not requiring biopsy (152 patients) or normal breasts (40 patients). This assumption was made on the findings of clinical and mammographic examinations and on the results of follow-up for not less than 12 months.

The diagnostic accuracies of clinical examination as recorded by one observer and of thermography and 70-mm. mammography as recorded by three observers in the confirmed cases are shown in Table I. Thermography and 70$\mathrm{mm}$. mammography are obviously relatively inaccurate compared with clinical examination in both benign and malignant conditions. There was considerable observer error between 
the three radiologists. This has been further defined in Table II, in which the accuracy of diagnosis in malignant disease has been calculated when all three radiologists agreed with the diagnosis and when at least one report of the three radiologists was correct. TABLE I.-Accuracy of Methods of Examination in 77 Patients with Cancer
of Breast and in 145 Patients with Histologically Proved Benign Disease of Breast. $A, B$, and $C$ Refer to the Three Radiologists

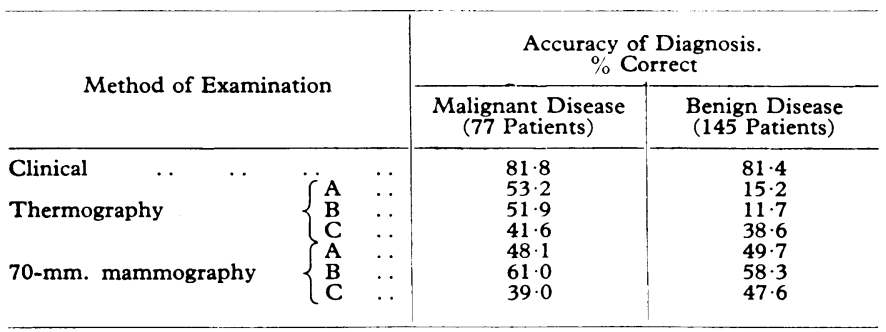

TABLE II.-Observer Error in Diagnosis in Patients (77) with Malignant Disease of Breast by Three Radiologists

\begin{tabular}{ll|c|c}
\hline & Thermography & $\begin{array}{c}\text { 70-mm. } \\
\text { Mammography }\end{array}$ \\
\hline All three radiologists correct & $\ldots$ & $29.9 \%$ & $31 \cdot 2 \%$ \\
All three radiologists wrong. & $\cdots$ & $32.5 \%$ & $28.6 \%$ \\
At least one radiologist correct & $\cdots$ & $67.5 \%$ & $71.4 \%$
\end{tabular}

The incidence of false-positive and false-negative reports with each method of investigation has been calculated for patients with histologically proved benign and malignant disease in the symptomatic group (Table III). There is a strikingly high incidence of false-negative diagnoses of cancer in patients with malignant disease. The false-positive rate in patients with benign disease is less pronounced.

TABLE III.-False-positive Rates (i.e., \% Suspected Malignant) in 145 Patients with Histologically Proved Benign Disease and False-negative Rates (i.e., \% Suspected Benign) in 77 Patients with Malignant Disease. $A, B$, and $C$ Refer to the Three Radiologists

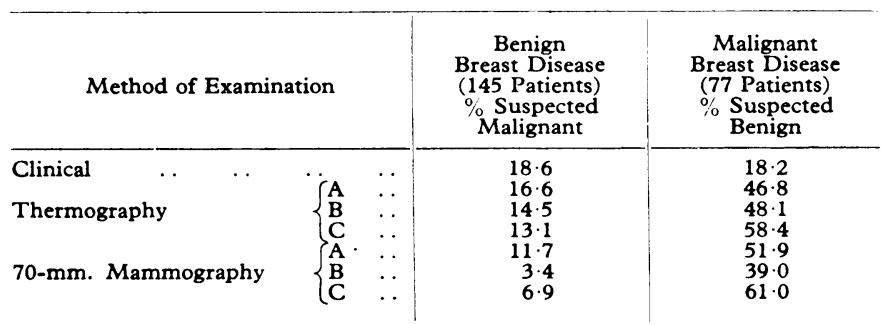

While the survey was carried out to determine specifically the value of each of the methods in the diagnosis of malignant disease, their accuracy in combination has also been considered in the 77 patients with cancer. The diagnostic accuracy of clinical examination alone, clinical examination plus thermography, clinical examination plus $70-\mathrm{mm}$. mammography, and all three methods combined are noted in Table IV. In this table a report of suspected malignancy by any one of the three radiologists was included as positive for that patient, and represents the best possible accuracy. TABLE IV.-Diagnostic Accuracy of Clinical Examination Alone and Com-
bined with Thermography and 70-mm. Mammography in 77 Patients with Cancer of Breast

\begin{tabular}{|c|c|c|}
\hline \multirow[t]{2}{*}{ Method of Examination } & \multicolumn{2}{|c|}{$\begin{array}{l}\text { Correct Diagnosis in } \\
\text { Malignant Disease. } \\
\text { (77 Patients) }\end{array}$} \\
\hline & No. & $\%$ \\
\hline 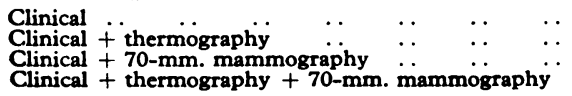 & $\begin{array}{l}63 \\
69 \\
70 \\
73\end{array}$ & $\begin{array}{l}81 \cdot 8 \\
89.6 \\
90.9 \\
94 \cdot 8\end{array}$ \\
\hline
\end{tabular}

The addition of thermography and 70-mm. mammography to clinical examination enhanced the accuracy of the diagnosis of malignancy. Thus one radiologist of the three (but not the same radiologist each time) correctly diagnosed cancer by thermography in six patients and by $70-\mathrm{mm}$. mammography in seven in whom the correct diagnosis was not made clinically. With the use of both methods to complement clinical examination, 10 patients with a clinical diagnosis of benign disease were suspected of having cancer. However, all these patients had a clearly palpable discrete lump in the breast and consequently would have been submitted to biopsy.

One of the reasons for the low accuracy of $70-\mathrm{mm}$. mammography in this survey was the high incidence of unsatisfactory films reported by each observer. The number of thermograms and $70-\mathrm{mm}$. mammograms in the symptomatic group considered not entirely satisfactory technically by each observer but still reportable is shown in Table $\mathrm{V}$ : almost half of the $70-\mathrm{mm}$. mammograms were of inferior quality (average $45.7 \%$ ) whereas $79 \%$ of thermograms were accepted as satisfactory. A similar proportion of $70-\mathrm{mm}$. mammograms in the asymptomatic group were reported as imperfect, and these poor quality films did not diminish in proportion as the survey proceeded.

TABLE V.-Thermograms and 70-mm. Mammograms Reported as Unsatisfactory by each Radiologist $(A, B$, and $C)$ in the Symptomatic Patients (414)

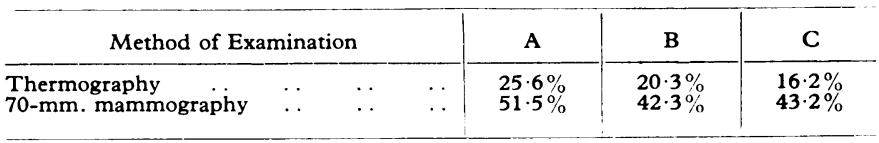

\section{Asymptomatic patients}

The results of surveying the 477 patients without symptoms of breast disease by clinical examination, thermography, and 70-mm. mammography are shown in Table VI. Clinical examination did not show any lesions which caused suspicion of malignant disease. Both thermography and 70-mm. mammography, however, detected suspicious lesions. When the findings of at least one observer for each patient were considered, $11.7 \%$ of the 477 patients were thought to have a malignant lesion on thermography and 2.5 on $70-\mathrm{mm}$. mammography. The comparable figures for suspect benign disease are also shown in Table VI. More patients were suspected of having benign disease according to all three methods of examination.

TABLE VI.-Lesions Suspected in 477 Patients without Symptoms of Breas Disease. $A, B$, and $C$ Refer to the Three Radiologists, and Combined Results Indicate Total Percentage when at Least One of the Three Radiologists Identified a Lesion

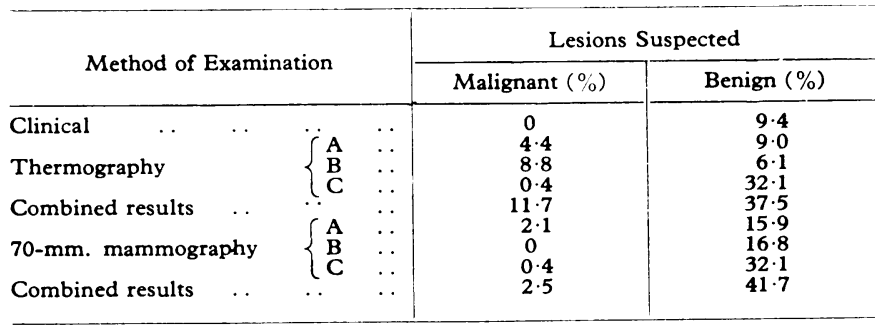

Again, there was considerable disagreement between the three radiologists concerned in reporting the thermograms and the $70-\mathrm{mm}$. mammograms. Though at least one observer noted malignant disease on thermography in 56 women, in only nine was there agreement between two observers and in only two between all three. In none of the $70-\mathrm{mm}$. mammograms reported as malignant did two or all three of the radiologists agree on a diagnosis.

The combined results of all three methods of investigation suggest that 67 women in the asymptomatic group had malig- 
nant disease of the breast and 307 had benign disease. These women with suspected malignancy were offered further investigation. If lesions were confirmed, the surgical opinion of a consultant was sought.

At the time of writing, 12 months after the end of the survey, biopsy had been carried out in 13 patients, in all of them proving benign. Eleven further patients in whom a repeat thermogram was again suspicious of malignancy were still under surveillance.

\section{Discussion}

The results of this survey indicate that the diagnostic accuracy of thermography and $70-\mathrm{mm}$. mammography does not approach that of clinical examination in patients with symptomatic breast disease. Thus, while one clinician correctly diagnosed malignant disease in $82 \%$ of women with breast complaints, the best possible accuracy, as represented by the highest individual accuracy when three radiologists independently surveyed the films, was only $53 \%$ for thermography and $61 \%$ for $70-\mathrm{mm}$. mammography. Even when one correct report from any of the three radiologists was considered, the accuracy was only $68 \%$ for thermography and $71 \%$ for $70-\mathrm{mm}$. mammography. Therefore neither thermography nor $70-\mathrm{mm}$ mammography is suitable for use as an isolated procedure in the diagnosis of breast disease. Nor can either method of examinaion be used in isolation as a screening procedure. Indeed, we consider it dangerous to use each method alone, as it would give the patient false security.

Furthermore, there is considerable observer error between radiologists using these two methods: thus all three radiologists agreed with the diagnosis in less than one-third of cases. A single radiologist reporting either thermogram or $70-\mathrm{mm}$. mammogram films is likely to be inaccurate in a significant proportion of cases. The inaccuracy of $70-\mathrm{mm}$. mammography is partly explained by the technical difficulty of getting films of satisfactory quality though possibly with improved technique it may be of more value. The use of these methods as complements to clinical examination improved the diagnostic rate, but in all the patients in whom this occurred a palpable lump was present.

The incidence of false-positive reports as assessed in patients with benign disease and asymptomatic women is high. This has led to a considerable expenditure of time and money for the further investigation of suspect patients. As the number of asymptomatic women studied was relatively small and many were young, it is not surprising that no cancers have come to light in this group.

There is a current tendency to promote screening programmes for breast cancer with a single method of examination. Many such programmes have been set up and are generally uncontrolled. The reports of the New York group indicate that neither clinical examination nor mammography alone is an adequate method of screening, and our survey indicates clearly that thermography and $70-\mathrm{mm}$. mammography have no place as isolated screening procedures.

We are grateful to Dr. Norval Taylor, Dr. Gillian Ford, and the late Dr. Michael Adams, of the D.H.S.S., for their interest; to the Department of Health and Social Security; and to Tenovus, Cardiff, for financing this study. We are also grateful to the consultants, especially those in obstetrics and gynaecology, Cardiff Royal Infirmary, for permission to survey patients under their care.

\section{REFERENCES}

Gravelle, I. H. (1969). Odelca Mirror, 8, 10.

Strax, P., and Oppenheim, A. (1968). American fournal of Roentgenology and Radium Therapy, 102, 941.

Strax, P., Shapiro, S., and Venet, L. (1968). In Prognostic Factors in Breast Cancer ed. A. P. M. Forrest and P. B. Kunkler, p. 242. Edinburgh, Livingstone.

Strax, P., Venet, L., Shapiro, S., and Gross, S. (1967). Cancer (Philadelphia), $20,2184$.

Strax, P., Venet, L., Shapiro, S., Gross, S., and Venet, W. (1969). Fournal of the American Medical Association, $210,433$.

Venet, L., Strax, P., Venet, W., and Shapiro, S. (1969). Cancer (Philadelphia), $24,1187$.

and its role in the local immune defence system of the respiratory, and gastrointestinal tracts has been shown by finding in those secretions naturally occurring antibodies of the $\operatorname{IgA}$ class to viruses and bacteria and by the viral neutralizing activity of human nasal secretions after poliovirus and influenza infections (Rossen et al., 1966; Bellanti et al., 1967; Bellanti, 1968; Tourville et al., 1968).

The recent finding of $\operatorname{IgA}(\mathrm{s})$ in normal human urine (Bienenstock and Tomasi, 1968) and the suggestion that urinary IgA may participate in local immune defence mechanisms led us to study the role of $\operatorname{IgA}(\mathrm{s})$ in non-obstructive chronic and recurrent urinary tract infections in children.

\section{Introduction}

In some body secretions such as tears, saliva, and those of the gastrointestinal tract, secretory $\operatorname{IgA}(\operatorname{IgA}(\mathrm{s}))$ is the predominant immunoglobulin (Chodirker and Tomasi, 1963; Tomasi and Zigelbaum, 1963). This IgA is chemically and immunologically distinct from serum IgA (Pollak et al., 1968),

\section{Patients and Methods}

The study included 29 patients (10 normal children, 17 with urinary tract infection (one a newborn), and two with ileal bladders). All patients with urinary tract infections had either chronic or recurrent (three or more) infections without evidence of significant anatomical or functional abnormalities as defined by intravenous pyelogram, cystourethrogram, cystoscopy, and creatinine clearance.

Specimens.-Twenty-four-hour urine samples were 